\title{
The Study of Relationship between Facial Width-to-Height Ratio and Aggression
}

\author{
Yaozhong Liu, Jia He \\ School of Management, Jinan University, Guangzhou, China \\ Email: 591861083@qq.com
}

Received 12 September 2015; accepted 5 October 2015; published 9 October 2015

Copyright (C) 2015 by authors and Scientific Research Publishing Inc.

This work is licensed under the Creative Commons Attribution International License (CC BY). http://creativecommons.org/licenses/by/4.0/

\begin{abstract}
Facial-width-to-height ratio (fWHR) is a kind of facial feature that based on the overall facial information and can be specifically measured. fWHR is closely related to the individual aggressive. The present study reviewed previous researches on aggression and measurement of fWHR, and described the relationship between fWHR and aggression under the external and internal factors. Moreover, the researchers explained them from the perspective of cognition, physics and evolutionary. In the future, the studies can deepen these fields to enrich the theories and effectively prevent and control violence in society.
\end{abstract}

\section{Keywords}

\section{fWHR, Aggression, Cognitive Neuroscience}

\section{Introduction}

As Parke and Slaby [1] said: "In the years of this century, a few number of topics, like aggression and control, there is so many theories and practice researches on them” [2]. As a complex social phenomenon, aggression has been the focus of academic attention.

Previous researches mainly depend on aggressive scales [3] [4] or the observation of life events [5] [6], these methods are vulnerable to the influence of subjective experience, generally with a strong sense of subjectivity. In recent years, with the prevalence of faces trait research, studies about face traits are deeper and deeper. Many researchers found, as a face trait which is independent to shape changing, fWHR (facial width-to-height ratio) is closely related to the individual's aggressiveness [7]-[9]. Studies have found that under the influence of internal and external factors, such as, different genders, ages, cultural backgrounds, stimulus materials, fWHR was still significantly associated with aggression (for example, Geniole, et al., 2012 [10]; Boshyan, Zebrowitz, Franklin, McCormick and Carré, 2013 [11]; Short, et al., 2012 [12]; Třebický, et al., 2015 [13]). Researchers introduced the variable-fWHR, and discussed the physical and social-psychological source of aggression from an entirely 
new perspective. Meanwhile, the findings of fWHR cognitive neuroscience provide a more adequate evidence to explain individual aggression mechanism, which is very important for society to prevent and control violence.

\section{2. fWHR and Aggression}

\section{1. fWHR's Concept and Its Measurement}

This concept of fWHR was first introduced in their study of gender characteristics of the face by Weston, Friday and Liò (2007) [14]. The fWHR is the ratio of the width and height of the face. The details of the calculation are: width is the distance between the left and right zygoma (face of the far left and far right) and height is the distance between upper lip and eyebrow (Alrajih and Ward, 2014 [15]; Dou, Liu and Zhang, 2014 [16]).

The measurement of fWHR is generally consistent with Carré and McCormick’s (2008) [6], which measured width and height of 2D front photos by ImageJ software, see Figure 1. In addition to using plane photos, some studies also measure fWHR by using 3D face scanning technology in order to avoid interference of measuring tools (Alrajih and Ward, 2014 [15]; Lefevre, et al., 2012 [17]; Kramer, Jones and Ward, 2012 [18]). Above both methods are indirect measurements, there is also another measurement technology-Anthropometry. Considering the relationship between body mass index (BMI) and fWHR, using anthropometry needs to control BMI before measurement. Anthropometry also needs to take photos from several angles like 2D and 3D measurement techniques, and then determine every point, finally use sliding calipers, gauges and other measuring tools to measure the height and width of the face (Kramer, Jones and Ward, 2012 [18]).

In a word, although fWHR is a static stability characteristic determined by the face bones, due to the different measurement methods and material samples, the characteristics perception of fWHR also varies.

\subsection{Aggression's Concept and Its Measurement}

Aggression, as a major cause of social violence (Bandura, 2001 [19]; Pan, 2005 [20]), has been the focus of scholars. Although the study of aggression has made rapid progress in last decades, there is still much disagreement in terms of its definition. There are mainly two different views. One view is that aggression is a goal-directed behavior referred to intentionally body attack or psychological damage (Berkowitz, 1993 [21]; Yang and Sun, 2010 [22]). Another view is that the aggression is a trait, showing the internal psychological characteristics of aggressive behavior. Because of physiological reasons, it may be that some people are born with high aggressiveness (Pan, 2005 [20]).

Direct reporting method is the most common method in study of relationship between fWHR and aggression, including other-report and self-report. Other-report methods refer to the subjects who make judgment about one's aggression according to the first impression of the face photo (Lefevre and Lewis, 2014 [9]; Short, et al., 2012 [12]; Carré \& McCormick, 2009 [23]); self-report methods refer to the subjects' own complete set of aggressive scale, experimenters get the aggressiveness level and take subject's photo samples, measuring the fWHR (Özener, 2012) [3]. Experimental method is practiced by certain experimental situations. The Point Subtraction (PASP) is more representative (Cherek, 1981) [24], and it is highly related to self-reported aggression level (Gerra, et al., 2007 [25]; Golomb, Cortez-Perez, Jaworski, Mednick and Dimsdale, 2007 [26]). After several

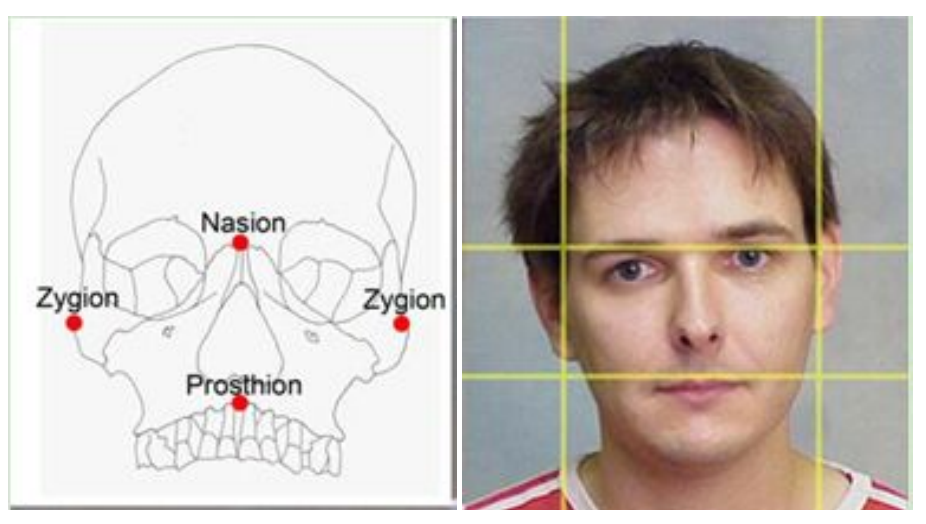

Figure 1. An example illustrating how the facial width-to-height ratio (FWHR) was calculated, taken from Weston et al., 2007. 
times of improvements (Carré and McCormick, 2008 [27]), its fixed pattern formation is as follows: the experimenter creates a competed atmosphere through instructions, and subjects believe that they need to compete withthe other subject in this atmosphere. There are three keys before participants: representing the reward, aggressive and protective action respectively, experimenter measures subject's press reaction. For those studies, it is difficult to carry out in the laboratory; researchers are more likely to use behavioral observation method, mainly by collecting actual behavioral data associated with the aggression. For example, Carré and McCormick [27] collected the number of penalty minutes per game obtained over Ontario University Athletics and the ESPN site as aggression indicator. Stirrat et al. (2012) [28] collected court cases that one die from exposure violence as part of its aggressive standards.

On the whole, past researches on aggression measurement mainly have two ways, one is through static questionnaire to measuring one's stable aggression personality tendencies indirectly, the other is through temporarily situation induced, or dynamic of faces expression (such as angry and anger) to rate one's aggression level. These researches all provide good supports for aggression behavior prevention. But someone directly measures a person's aggression through an objective, static fixed faces characteristic-fWHR, which is a new and effective way researchers have discovered in recent years.

\section{The Relationship between fWHR and Aggression}

The discussion of relationship between face feature and aggressive originally comes from the anthropologist's observation and analysis of primitive tribe, they found that male hunters with more violent aggressive behaviors have more wider face (a component of fWHR) than who without violent aggressive behaviors (Christiansen and Winkler, 1992 [29]). But psychologists haven't concerned fWHR, the particular facial features at this time, and did not pay more attention to the close relationship between it and aggression. Until Weston et al. (2007) [14] firstly proposed the concept of fWHR, the researchers began to study the relationship between fWHR and aggression.

Carré and McCormick (2008) [6] used two experiments to verify the relationship between college hockey player and national professional hockey player's fWHR and aggression. The evaluation of photos from subjects is as the subjective indicator of aggression. The number of penalty minutes per game for athlete as objective indicators of aggression. The results showed that fWHR is significantly associated with aggression. Afterwards, Carré et al. (2009) [23] made further research on both relationship, experimenters showed 37 male faces' pictures to subjects with 39 - $2000 \mathrm{~ms}$ random interval in experiment, and asked participants to make subjective evaluation of aggression in 7-point scale (1: not aggressive; 7: very aggressive), results showed that regardless of faces pictures rendering speed, aggressive and fWHR are significantly related. In addition, Carré and McCormick (2010) [30] came to the same conclusion by comparing face aggressive in two different experimental conditions (fWHR highlighted, weakening other facial features and weakening fWHR, emphasizing other facial features).

\subsection{The Effect on Relationship between fWHR and Aggression by Internal Factors Such as Gender and Age}

Previous studies mostly used male faces as stimuli, and found fWHR is significantly associated with aggression. Geniole, Keyes, Mondloch, Carré, and McComick (2012) [10] used the women’s faces as stimulus, subjects were asked to evaluate their aggression, results showed that the relationships between fWHR and aggression also apply to female faces, further evidences proved the stability of relationship between them.

In addition, the age differences influence the ability of emotion regulation and individual perception, and also influence the faces information process. Studies have found that elderly participants in the faces of information are more likely to be processed as positive information (Castle, et al., 2012 [31]; Ruffman, Sullivan, \& Edge, 2006 [32]; Zebrowitz, Franklin, Hillman, \& Boc, 2013 [33]). Boshyan, Zebrowitz, Franklin, McCormick, and Carré (2013) [11] respectively selected 56 young subjects (average age 26) and 51 old subjects (average age 75.6), used validated pictures of Carré et al. (2009) [23] as experiment material, and then compared the difference in different age subjects on the perception of the relationship between the two. Results showed that while elderly had a lot of differences in visual skills, mood and cognitive function with young people, the perception of faces was as sensitive as young man, which also showed the perception of aggression was an automated 
process. Although people of different ages have some differences in making use of this clue, fWHR remains an effective clue applied to different age groups during the process of discriminating face aggression automatically.

\subsection{The Effect on Relationship between fWHR and Aggression by Different Cultural Contexts}

Carré's series of studies showed that as a facial feature, fWHR was an effective clue in judging aggression, but there was a big difference in different race's facial features across the world, so did cultural background may affect the relationship between fWHR and aggression? Short et al. [12] selected 24 white neutral expressions faces from Canada Bruner University and 24 Zhejiang yellow neutral expressions faces from Sci-Tech University as stimulus, measured these face's fWHR (Weston, et al., 2007) [14], and asked adult and the children to evaluate the faces of different races. In order to ensure each child have consistency understanding of the aggression, experimenter gave them a simple description and training before the formal experiment. Results of the two groups of subjects showed that the perceptive judgment of aggression are significantly correlated with fWHR itself .But there are some inconsistent conclusion, previous studies found that the adults' perception of structure was weaker than child's (Mondloch, et al., 2010 [34]; Rhodes, et al., 2006 [35]), where adults and children on the judging relationship between fWHR and aggression has a high consistency. This result suggests that although cultural differences will have a major impact on cognitive differences, as a stable physiological indicator, fWHR perception have cross-cultural consistency.

\subsection{The Effect on Relationship between FWHR and Aggression by Stimulus Difference}

The effectiveness of stimulus material is an important factor influencing the results of the experiment; the same experimental design due to differences in stimulus might come to a different conclusion. Above studies selected the average person faces as stimuli, demonstrated fWHR highly correlated with aggression. If it use photo of groups with high aggressive as stimulus, it will be similar to the conclusion? Třebický et al. (2013) [36] chose mixed-martial-arts fighters as the object of study, selected 146 fighters' photos from the ultimate fighting Championship (UFC) as experimental materials, took subject's aggression evaluation as subjective indicators of aggression and the frequency of attacking as objective indicators of aggression. fWHR is not measured by software directly, rather than by geometric morphometric techniques (GMM: a form of statistical methods)in this study. Especially experimenter made spatial analysis in 72 face points to determine which specific morphological characteristics influence the evaluation of aggression. Final GMM result showed better professional fighter had wider face, which further revealed the fWHR was an effective clue to inferring aggression, and a useful indicator to predict fighter performance. Třebický, Fialová, Kleisner, Roberts, Little and Havlíček (2014) [13] continued to expand this research, they take fighter's height and weight as control variables to analysis. Results showed fWHR was positively correlated with the players fighting skills, also indicated that the perception of aggression was related to one's actual aggression.

In addition, some researchers have selected a particular facial feature to make comparative study. Geniole, Molnar, Carré and McCormick (2014) [37] take several experiments, they chose faces with beard and faces without beard as stimulus material, respectively measured these fWHR (Carré and McCormick, 2008) [6], and then asked subjects make evaluation about aggression and trust during the picture shown in different speed, last but not least, made statistics analysis on these data. The findings showed the link between fWHR and aggression were more directly and closely, and it was faster to make aggression judgments of face than make trust judgments.

Geniole and McCormick (2015) [8] discussed the above issues again; they made a longitudinal study instead of comparative study this time. They searched for people who had token photos to record their beard growth over a long period of time, and selected some pictures that before and after grow beard as final stimulus material, including totally 50 photos belonging to 25 peoples. After that, experimenter asked subjects to make evaluation about aggression and attractive according to first impression, and measured fWHR of every photos. Statistical analysis showed that fWHR was significantly correlated in case with or without beard, there was no significant differences, and the assessment are highly relevant in both cases, without being affected by beard.

Above all studies were taken the real photos of people as stimulus material directly, so that there may be other facial features except the fWHR characteristics influencing subject's perception and evaluation. Lefever and Lewis (2014) [9] according to the purpose of study, they took two experiments. In experiment 1, they synthe- 
sized a total of 72 fWHR different photos $(12 \times 2 \times 3)$ with twelve individual composite identities combined by three images of Caucasian men in two size fWHR prototypes, according to $\pm 25 \%, 37.5 \%$ and $50 \%$ three levels of transformation. Show subjects these photos in pair after precisely such controlled, and allow participants to choose one looked more aggressive. Final results showed that fWHR was significantly related to aggression, there are significant differences even the subtle changes of face information (25\% difference in fWHR). In experiment 2, they synthesized photo as the same way in experiment 1 with Caucasian woman and other experimental procedures remain unchanged. The final result was in accordance with Geniole et al. (2012) [10] study the fWHR of woman was also significantly correlated with aggression.

These studies proved that fWHR was an effective clue to predict aggression, but there were some still researches inconsistent with these conclusions. Deaner, Goetz, Shattuck and Schnotala (2012) [5] collected all of the NHL (National Hockey League) player information as research material, found that the weight of athlete can predict their aggressive behavior very well, not the fWHR. Similar to this, Özener (2012) [3] take Turkey as research object, taken their pictures, test fWHR and finish an aggression scale to aggression score. Results found that there was no significant relationship between FWHR and self-reported aggression score. Gómez-Valdés et al. (2013) [38] collected 4960 subjects' information from 94 countries, including a number of ethnographic records and crime personnel's information; they came to a conclusion through the analysis of these documents that the fWHR of human had no significant relationship with their aggressive behavior.

There are any inconsistency among these research conclusion, it perhaps because researchers take different studying object and different research method. In addition, as an integrated character, aggression will also be affected by environmental effects, such as family and social setting. A lot of research results show that the fWHR is important clues to determine one's aggression. It was remains researchers to make further study to determine the perceptual judgment ability with fWHR was derived from birth in the process of human evolution, or acquired from growth experience, or both.

\section{The Theoretical Explanation of the Relationship between fWHR and Aggression}

Theoretical explanations of relationship between the two mainly consists of three levels, one is the level of human evolution (Carré and McCormick, 2008 [6]; Stirrat, Stulp, and Pollet, 2012 [28]; Sell, et al., 2009 [39]), the second is the levels of physiological estrogen (Mazur and Booth, 1998) [40], the third is the level of cognitive neuroscience (Carré, Murphy and Hariri, 2013) [41]. These theoretical explanations are not exclusive mutually but mutually advancement and promoting.

\subsection{Evolutionary Anthropology}

Judging from the long human evolution history, there is a great sense for human survival to recognize aggression. In order to make decisions of face the battle or evasion to ensure their own survival, it's important to estimate the competitor's fighting force and aggression in advance. Studies have found that making judgment on aggression and threaten about face was faster than on intellectual (Bar, Neta and Linz, 2006) [42] and trust (Geniole, Molnar, Carré and McCormick, 2014) [37] judgment. From the perspective of evolutionary psychology, angry facial expression was consist of raising the upper lip and lowering the brow, the pattern of muscle activity will naturally make the facial width to height ratio increases (Carré and McCormick, 2008) [6]. At times of resource competition, it is critical to identify negative emotions quickly to ensure their survival and reproduction (Sell, et al., 2009) [39]. So we may conclude that cultivating the sensitivity to fWHR is to avoid physical attacks and gain resource in the evolution of mankind. People with wider face will be more aggressive and dangerous in combat situations, and there may be more deaths due to violence (Stirrat, Stulp and Pollet, 2012) [28].

Explaining the relationship between fWHR and aggression from the perspective of evolutionary psychology has its theoretical advantages, but evolutionary psychology could not repeats history itself, and explore the relationship between the two under controlling related variables from the perspective of empirical, so there are no direct, strong evidence to prove the relationship of fWHR and aggression is the result of human evolution.

\subsection{The Physiological Hormone Level}

The first theoretical explanation of relationship between fWHR and aggressive is that levels of testosterone af- 
fect secondary sex characteristics at puberty, testosterone release of adolescent male influences the growth of male skulls (Carré, McCormick and Hariri, 2011) [43]. Studies have shown that the male fWHR and base levels of testosterone was correlated, and levels of testosterone and aggression was also correlated (Mazur and Booth, 1998). Lefevre et al. (2013) [44] measured 185 men’s testosterone levels before and after activity in speed dating activity, and tested their fWHR. Results showed fWHR was positively correlated baseline testosterone level and after activation levels, indicating that the relationship between testosterone and fWHR was the internal mechanisms of .fWHR and aggression.

Although the physiological hormone levels is a more objective physiological indicator, whether testosterone levels can influence the fWHR size and aggression or aggression changes affect the release of testosterone, then affects the fWHR size. Current researches on these three contextual links without a clear explanation, and which need further studies to explore.

\subsection{Cognitive Neuroscience Mechanism}

Confirming basic neurophysiology mechanism of aggression plays a major role to reduce and prevent aggressive behavior. Animal studies have shown that aggression was related to the activity of the neural circuitry comprising the periaqueductal gray matter (PAG), hypothalamus and amygdala (Siegel, et al., 2007) [45]. Human brain imaging studies have also found that amygdala activity would enhance when individuals at risk and take aggressive behavior (Coccaro, et al., 2007 [46]; Beaver, et al., 2008 [47]; Manuck, et al., 2010 [48]). The series of studies show that the amygdala is the neural connection to adjust aggression and social signals (Carré, et al., 2011) [43]. In view of this, Carré, Murphy and Hariri [41] used fMRI (functional magnetic resonance imaging) technology to study the relationship between brain activity, fWHR and aggression. The study measured 64 subjects' fWHR, and evaluated their aggression by BPAQ scale (Buss-Perry Aggression aggressive scale Questionnaire). In fMRI paradigm, participants watch a trio of faces and select one of two faces looked more aggressive during face-match, and made choice from a trio of simple geometric shapes during sensorimotor control blocks. Correlation analysis showed fWHR and sex regulated the amygdala activity and aggressive behavior. Male right amygdala activity and fWHR significantly related $\left(\delta \mathrm{r}^{2}=30.8 \%, \mathrm{p}<0.01\right)$; when the fWHR was big, right amygdala activity and aggressiveness scores have significant positive correlation $(\beta=8.34, \mathrm{p}<0.01)$, whereas fWHR is small, no significant correlation between the two $(\beta=-4.06, \mathrm{p}>0.01)$. For male subjects in this study, big fWHR increased amygdala activities when facing interpersonal threat signal (angry expression) which predicted differences of personal aggression, small fWHR didn’t have this effect. Researchers (Carré, Murphy and Hariri [41]) also found that aggression and amygdala activity only was related in the face of angry expression, and not of fear, surprise and neutral facial expressions. This was similar to previous clinical studies on patients with intermittent manic that only angry expressions caused their dramatic activity in amygdala among patients often occurred aggressive behavior (Coccaro, et al., 2007) [46]. This also suggested that there was a direct relationship between anger and aggressive behavior, provided neurophysiology evidence of the relationship between fWHR and aggression.

To sum up, according to current research, fWHR, as an non-verbal signal to infer aggression, can cause activation of the neural network in certain areas, including anger, threats and other negative stress related brain area and the offensiveness itself corresponds to areas of the brain, which plays a significant role in concluded specific cognitive neuroscience of relationship between the two.

\section{Conclusions}

Since Weston et al. (2007) [14] presented the concept of fWHR for the first time, a large number of studies have shown that fWHR was closely related to human aggression, the people with big fWHR were more aggression and vice versa. Meanwhile, fWHR, as a new clue, has important implications and promotion to the aggression research. However, this relationship is not completely absolute, the study of the relationship between the two is only at a preliminary stage, there are a lot of issues which have not been resolved in this area, which is needed to further explore in future research.

In most of the studies mentioned above, self-report method were used in measurements of aggression, few studies took PASP laboratory method to measure aggression, and in some studies, researchers took daily events to judge a person's aggression. But in these three evaluation methods in addition to the internal validity of the 
issue, external promotion validity is to be verified. How future research uses multidimensional indicators to assess the aggression also needs to take into consideration.

Current study only finds that the perception of relationship between fWHR and aggression is only connected with amygdala (Carré, Murphy and Hariri) [41]. With the constant development of cognitive neural technology, there will be more and more multiple methods to make deeper study on this problem, including the recognition of fWHR, the evaluation of aggression, the perception of the relationship between the two and the whole process. It is very significant for us to further explore these series of problem to deeper understand the relationship between them.

In addition, studies on the relationship between the two are almost in the context of western culture, and select a particular group as subjects. However, is it consistent with the researches in west countries on perception of face features in those countries' cultural backgrounds which is different from the western culture? The studies in China with 5000 years of culture are far few. China is a country deeply influenced by Confucian culture, emphasizing the "Harmony is precious", the doctrine of mean thought, so people tend to avoid conflict and aggression, and to solve the problem in a peaceful way. In this cultural context whether people evaluate aggression of face depends on the fWHR clue, and what is the difference between east and west culture on perception of aggression needs further research to continue to verify.

In this article, we introduced the relationship between fWHR and aggression by many interesting studies, and explained this relationship from different levels, including cognitive neuroscience. Importantly, we have proposed a new perspective for further study of aggression, which has great inspiration for theory and practice research.

\section{References}

[1] Parke, R. and Slaby, R. (1983) The Development of Aggression. In: Mussen, P. and Hetherington, E., Eds., Handbook of Child Psychology: Socialization, Personality, and Social Development, Vol. 4, Wiley, New York, 457-641.

[2] Zhang, X.Y., Gao, D.G. and Fu, H. (2011) Dialectical Thinking Reduces Aggressive Tendencies. Acta Psychologica Sinica, 43, 42-51. http://dx.doi.org/10.3724/SP.J.1041.2011.00042

[3] Özener, B. (2012) Facial Width-to-Height Ratio in a Turkish Population Is Not Sexually Dimorphic and Is Unrelated to Aggressive Behavior. Evolution and Human Behavior, 33,169-173. http://dx.doi.org/10.1016/j.evolhumbehav.2011.08.001

[4] Buss, A.H. and Perry, M. (1992) The Aggression Questionnaire. Journal of Personality and Social Psychology, 63, 452-459. http://dx.doi.org/10.1037/0022-3514.63.3.452

[5] Deaner, R.O., Goetz, S.M.M., Shattuck, K. and Schnotala, T. (2012) Body Weight, Not Facial Width-to-Height Ratio, Predicts Aggression in Pro Hockey Players. Journal of Research in Personality, 46, 235-238.

http://dx.doi.org/10.1016/j.jrp.2012.01.005

[6] Carré, J.M. and McCormick, C.M. (2008) In Your Face: Facial Metrics Predict Aggressive Behaviour in the Laboratory and in Varsity and Professional Hockey Players. Proceedings of the Royal Society of London: Series B Biological Sciences, 275, 2651-2656. http://dx.doi.org/10.1098/rspb.2008.0873

[7] Haselhuhn, M.P., Ormiston, M.E. and Wong, E.M. (2015) Men’s Facial Width-to-Height Ratio Predicts Aggression: A Meta-Analysis. PLoS ONE, 10, e0122637. http://dx.doi.org/10.1371/journal.pone.0122637

[8] Geniole, S.N. and McCormick, C.M. (2015) Facing Our Ancestors: Judgements of Aggression Are Consistent and Related to the Facial Width-to-Height Ratio in Men Irrespective of Beards. Evolution and Human Behavior, 36, $279-285$. http://dx.doi.org/10.1016/j.evolhumbehav.2014.12.005

[9] Lefevre, C.E. and Lewis, G.J. (2014) Perceiving Aggression from Facial Structure: Further Evidence for a Positive Association with Facial Width-to-Height Ratio and Masculinity, but Not for Moderation by Self-Reported Dominance. European Journal of Personality, 28, 530-537.

[10] Geniole, S.N., Keyes, A.E., Mondloch, C.J., Carré, J.M. and McCormick, C.M. (2012) Facing Aggression: Cues Differ for Female versus Male Faces. PLoS ONE, 7, e30366. http://dx.doi.org/10.1371/journal.pone.0030366

[11] Boshyan, J., Zebrowitz, L.A., Franklin, R.G., McCormick, C.M. and Carré, J.M. (2013) Age Similarities in Recognizing Threat from Faces and Diagnostic Cues. The Journals of Gerontology. Series B, Psychological Sciences and Social Sciences, 69, 710-718.

[12] Short, L.A., Mondloch, C.J., McCormick, C.M., Carré, J.M., Ma, R., Fu, G. and Lee, K. (2012) Detection of Propensity for Aggression Based on Facial Structure Irrespective of Face Race. Evolution and Human Behavior, 33, 121-129. http://dx.doi.org/10.1016/j.evolhumbehav.2011.07.002 
[13] Třebický, V., Fialová, J., Kleisner, K., Roberts, S.C., Little, A.C. and Havlíček, J. (2015) Further Evidence for Links between Facial Width-to-Height Ratio and Fighting Success: Commentary on Zilioli et al. (2014). Aggressive Behavior, 41, 331-334.

[14] Weston, E.M., Friday, A.E. and Liò, P. (2007) Biometric Evidence that Sexual Selection Has Shaped the Hominin Face. PLoS One, 2, e710. http://dx.doi.org/10.1371/journal.pone.0000710

[15] Alrajih, S. and Ward, J. (2014) Increased Facial Width-to-Height Ratio and Perceived Dominance in the Faces of the UK’s Leading Business Leaders. British Journal of Psychology, 105, 153-161. http://dx.doi.org/10.1111/bjop.12035

[16] Dou, D.H., Liu, X.C. and Zhang, Y.J. (2014) Babyface Effect: Babyface Preference and Overgeneralization. Advances in Psychological Science, 22, 760-771.

[17] Lefevre, C.E., Lewis, G.J., Bates, T.C., Dzhelyova, M., Coetzee, V., Deary, I.J. and Perrett, D.I. (2012) No Evidence for Sexual Dimorphism of Facial Width-to-Height Ratio in Four Large Adult Samples. Evolution and Human Behavior, 33, 623-627. http://dx.doi.org/10.1016/j.evolhumbehav.2012.03.002

[18] Kramer, R.S., Jones, A.L. and Ward, R. (2012) A Lack of Sexual Dimorphism in Width-to-Height Ratio in White European Faces Using 2D Photographs, 3D Scans, and Anthropometry. Plos ONE, 7, e42705. http://dx.doi.org/10.1371/journal.pone.0042705

[19] Bandura, A. (2001) Social Cognitive Theory: An Agentic Perspective. Annual Review of Psychology, 52, 1-26. http://dx.doi.org/10.1146/annurev.psych.52.1.1

[20] Pan, Q.M. (2005) Adolescent Aggressiveness: Dimension, Structure and Correlation Factors. Master's Thesis, South China Normal University, Guangzhou.

[21] Berkowitz, L. (1993) Aggression: It’s Causes, Consequences, and Control. McGraw-Hill, New York.

[22] Yang, Z.L. and Sun, L.R. (2010) Laboratory Paradigms on Aggression Studies. Psychological Science, 33, $1436-1438$.

[23] Carré, J.M., McCormick, C.M. and Mondloch, C.J. (2009) Facial Structure Is a Reliable Cue of Aggressive Behavior. Psychological Science, 20, 1194-1198. http://dx.doi.org/10.1111/j.1467-9280.2009.02423.x

[24] Cherek, D.R. (1981) Effects of Smoking Different Doses of Nicotine on Human Aggressive Behaviour. Psychopharmacology, 75, 339-345. http://dx.doi.org/10.1007/BF00435849

[25] Gerra, G., Zaimovic, A., Raggi, M.A., Moi, G., Branchi, B., Moroni, M. and Brambilla, F. (2007) Experimentally Induced Aggressiveness in Heroin-Dependent Patients Treated with Buprenorphine: Comparison of Patients Receiving Methadone and Healthy Subjects. Psychiatry Research, 149, 201-213. http://dx.doi.org/10.1016/j.psychres.2006.02.013

[26] Golomb, B.A., Cortez-Perez, M., Jaworski, B.A., Mednick, S. and Dimsdale, J. (2007) Point Subtraction Aggression Paradigm: Validity of a Brief Schedule of Use. Violence and Victims, 22, 95-103. http://dx.doi.org/10.1891/vv-v22i1a006

[27] Carré, J.M. \& McCormick, C.M. (2008) Aggressive Behavior and Change in Salivary Testosterone Concentrations Predict Willingness to Engage in a Competitive Task. Hormones and Behavior, 54, 403-409. http://dx.doi.org/10.1016/j.yhbeh.2008.04.008

[28] Stirrat, M., Stulp, G. and Pollet, T.V. (2012) Male Facial Width Is Associated with Death by Contact Violence: Narrowfaced Males Are More Likely to Die from Contact Violence. Evolution Human Behavior, 33, 551-556. http://dx.doi.org/10.1016/j.evolhumbehav.2012.02.002

[29] Christiansen, K. and Winkler, E.M. (1992) Hormonal, Anthropometrical, and Behavioral Correlates of Physical Aggression in Kung San Men of Namibia. Aggressive Behavior, 18, 271-280. http://dx.doi.org/10.1002/1098-2337(1992)18:4<271::AID-AB2480180403>3.0.CO;2-6

[30] Carré, J.M., Morrissey, M.D., Mondloch, C.J. and McCormick, C.M. (2010) Estimating Aggression from Emotionally Neutral Faces: Which Facial Cues Are Diagnostic? Perception, 39, 356-377. http://dx.doi.org/10.1068/p6543

[31] Castle, E., Eisenberger, N.I., Seeman, T.E., Moons, W.G., Boggero, I.A., Grinblatt, M.S. and Taylor, S.E. (2012) Neural and Behavioral Bases of Age Differences in Perceptions of Trust. Proceedings of the National Academy of Sciences of the United States of America, 109, 20848-20852. http://dx.doi.org/10.1073/pnas.1218518109

[32] Ruffman, T., Sullivan, S. and Edge, N. (2006) Differences in the Way Older and Younger Adults Rate Threat in Faces but Not Situations. The Journals of Gerontology, Series B: Psychological Sciences and Social Sciences, 61, 187-194. http://dx.doi.org/10.1093/geronb/61.4.P187

[33] Zebrowitz, L.A., Franklin Jr., R.G., Hillman, S. and Boc, H. (2013) Older and Younger Adults’ First Impressions from Faces: Similar in Agreement but Different in Positivity. Psychology \& Aging, 28, 202-212. http://dx.doi.org/10.1037/a0030927

[34] Mondloch, C.J., Elms, N., Maurer, D., Rhodes, G., Hayward, W.G., Tanaka, J.W. and Zhou, G.M. (2010) Processing 
Underlying the Cross-Race Effect: An Investigation of Holistic, Featural, and Relational Processing of Own-Race and Other-Race Faces. Perception, 39, 1065-1085. http://dx.doi.org/10.1068/p6608

[35] Rhodes, G., Hayward, W.G. and Winkler, C. (2006) Expert Face Coding: Configural and Component Coding of OwnRace and Other-Race Faces. Psychonomic Bulletin \& Review, 13, 499-505. http://dx.doi.org/10.3758/BF03193876

[36] Třebický, V., Havlíček, J., Roberts, S.C., Little, A.C. and Kleisner, K. (2013) Perceived Aggressiveness Predicts Fighting Performance in Mixed-Martial-Arts Fighters. Psychological Science, 24, 1664-1672. http://dx.doi.org/10.1177/0956797613477117

[37] Geniole, S.N., Molnar, D.S., Carré, J.M. and McCormick, C.M. (2014) The Facial Width-to Height Ratio Shares Stronger Links with Judgments of Aggression Than with Judgments of Trustworthiness. Journal of Experimental Psychology: Human Perception and Performance, 40, 1526-1541. http://dx.doi.org/10.1037/a0036732

[38] Gómez-Valdés, J., Hünemeier, T., Quinto-Sánchez, M., Paschetta, C., de Azevedo, S., González, M.F., et al. (2013) Lack of Support for the Association between Facial Shape and Aggression: A Reappraisal Based on a Worldwide Population Genetics Perspective. PLoS ONE, 8, e52317. http://dx.doi.org/10.1371/journal.pone.0052317

[39] Sell, A., Cosmides, L., Tooby, J., Sznycer, D., Von Rueden, C. and Gurven, M. (2009) Human Adaptations for the Visual Assessment of Strength and Fighting Ability from the Body and Face. Proceedings of the Royal Society B: Biological Sciences, 276, 575-584. http://dx.doi.org/10.1098/rspb.2008.1177

[40] Mazur, A. and Booth, A. (1998) Testosterone and Dominance in Men. Behavioral and Brain Sciences, 21, $353-363$. http://dx.doi.org/10.1017/s0140525x98001228

[41] Carré, J.M., Murphy, K.R. and Hariri, A.R. (2013) What Lies Beneath the Face of Aggression. Social Cognitive and Affective Neuroscience, 8, 224-229. http://dx.doi.org/10.1093/scan/nsr096

[42] Bar, M., Neta, M. and Linz, H. (2006) Very First Impressions. Emotion, 6, 268-278. http://dx.doi.org/10.1037/1528-3542.6.2.269

[43] Carré, J.M., McCormick, C.M. and Hariri, A.R. (2011) The Social Neuroendocrinology of Human Aggression. Psychoneuroendocrinology, 36, 935-944. http://dx.doi.org/10.1016/j.psyneuen.2011.02.001

[44] Lefevre, C.E., Lewis, G.J., Perrett, D.I. and Penke, L. (2013) Telling Facial Metrics: Facial Width Is Associated with Testosterone Levels in Men. Evolution and Human Behavior, 34, 273-279. http://dx.doi.org/10.1016/j.evolhumbehav.2013.03.005

[45] Siegel, A., Bhatt, S., Bhatt, R. and Zalcman, S.S. (2007) The Neurobiological Bases for Development of Pharmacological Treatments of Aggressive Disorders. Current Neuropharmacology, 5, 135-147. http://dx.doi.org/10.2174/157015907780866929

[46] Coccaro, E.F., McCloskey, M.S., Fitzgerald, D.A. and Phan, K.L. (2007) Amygdala and Orbitofrontal Reactivity to Social Threat in Individuals with Impulsive Aggression. Biological Psychiatry, 62, 168-178. http://dx.doi.org/10.1016/j.biopsych.2006.08.024

[47] Beaver, J.D., Lawrence, A.D., Passamonti, L. and Calder, A.J. (2008) Appetitive Motivation Predicts the Neural Response to Facial Signals of Aggression. Journal of Neuroscience, 28, 2719-2725. http://dx.doi.org/10.1523/JNEUROSCI.0033-08.2008

[48] Manuck, S.B., Marsland, A.L., Flory, J.D., Adam Gorka, A., Robert E. Ferrell, R.E. and Ahmad R. Hariri, A.R. (2010) Salivary Testosterone and a Trinucleotide (CAG) Length Polymorphism in the Androgen Receptor Predict Amygdala Reactivity in Men. Psychoneuroendocrinology, 35, 94-104. http://dx.doi.org/10.1016/j.psyneuen.2009.04.013 